\title{
HST MID-UV IMAGING OF NEARBY GALAXIES
}

\author{
Rogier A. Windhorst, Violet A. Taylor, and Rolf A. Jansen \\ Department of Physics and Astronomy, Arizona State University, Box 871504, Tempe, AZ 85287
}

\begin{abstract}
We summarize Hubble Space Telescope projects that imaged a significant sample of nearby galaxies of all types and inclinations in the mid-UV with WFPC2 and some in the near-IR with NICMOS. The sample emphasizes high-surface brightness late-type, irregular, peculiar and merging galaxies, since these objects are the most prevalent at high redshifts. The sample is used to establish a local benchmark for quantitative classifications of galaxies seen by HST in their rest-frame UV, and in the next decade also by the James Webb Space Telescope.

We review how nearby galaxies change their rest-frame structure and morphology from the rest-frame UV to the optical as a function of galaxy type and inclination, and other studies that are being done with this HST sample. When observed in the rest-frame mid-UV — early- to mid-type galaxies are more likely misclassified as later types than late-type galaxies are misclassified as earlier types. This is because later-type galaxies are dominated by the same young and hot stars in all filters from the mid-UV to the red, and so have a smaller "morphological K-correction" than true earlier type galaxies. This $K_{\text {mor } p h}$ can explain a small part, but certainly not all of the excess faint blue late-type galaxies seen in deep HST fields. Contrary to early-mid-type galaxies, the majority of late-type galaxies become redder outward. While young UV/blue-bright stellar populations dominate their inner morphology, most late-type galaxies are inferred to have a significant halo or (thick) disk of older stars. We discuss these results in a cosmological context.
\end{abstract}

Keywords: galaxies: elliptical — spiral — irregular — mergers — peculiar — ultraviolet

\section{The Need to Reliably Classify Faint Optical Samples}

Since the mid 1990's, faint galaxies have been observed systematically with the Hubble Space Telescope (HST). The best statistics, spatial sampling, and areal coverage are achieved in the red, and good HST structural information is available today for $\gtrsim 10^{5}$ galaxies with $\mathrm{I} \sim 26-27 \mathrm{mag}$. These galaxies come from the two Hubble Deep Fields ("HDF"s; Williams et al. 1996, 1998), the UltraDeep Field (UDF; Beckwith et al. 2004), their flanking fields, the HST Medium-Deep Survey, and other HST parallel surveys (e.g., Griffiths et al. 1994; Driver et al. 1995a; Abraham et al. 1996, 1999; Roche et al. 1997). The median half-light radius of faint field galaxies with $\mathrm{I} \sim 26$ mag is $r_{e} \simeq 0.2-$ 
0.3" (Odewahn et al. 1996, Cohen et al. 2003). HST's $2.4 \mathrm{~m}$ aperture limits the useful wavelength for proper galaxy classification to $4000 \lesssim \lambda \lesssim 8000 \AA$, because of the high spatial resolution and good sampling required. HST can determine morphology and structure with relative ease for a very large number of galaxies, and so explore a part of parameter space that constrains galaxy formation and evolution, which is complementary to spatially resolved spectroscopy and kinematics (see Koo 2004 and Le Fevvre 2004; this Vol.).

The most dramatic result from these HST studies was that at faint fluxes, late-type/irregular galaxies dominate the faint blue galaxy counts (Driver et al. 1995a; Glazebrook et al. 1995; Odewahn et al. 1996; Windhorst et al. 1997, 2000). These are believed to be star-forming galaxies that underwent substantial evolution since $\mathrm{z} \approx 3-4$ (Ellis et al. 1996; Pascarelle et al. 1996; Abraham et al. 1999). Ellipticals and early-type spirals evolved much less since $\mathrm{z} \lesssim 1$ (Driver et al. 1995a; Lilly et al. 1998; Cohen et al. 2003). Driver et al. (1998) suggested differential evolution of galaxies as a function of type, implying a gradual formation of the Hubble sequence with cosmic time.

\section{The Morphological K-correction and How to Address it}

In the deepest $H S T$ fields, where reliable classifications are achievable to $\mathrm{I} \leqslant 26$ mag (e.g., Odewahn et al. 1996; Driver et al. 1998), the sampled redshift range is $\mathrm{z} \simeq 1-3$, while the bulk of the galaxies is at $\mathrm{z} \sim 2$. Faint high redshift galaxies observed in the $I$-band are therefore primarily seen in the rest-frame mid-ultraviolet (mid-UV), or 2000-3200 $\AA$, which primarily traces high surface brightness ("SB") regions populated by high densities of young $(\lesssim 1 \mathrm{Gyr}$ ) hot stellar populations. The reliability of results derived from faint $H S T$ galaxy morphology is therefore fundamentally limited by the uncertain rest-frame mid-UV morphology of nearby galaxies. Although these faint, latetype/irregular objects resemble some classes of nearby late-type and peculiar galaxies (e.g., Hibbard \& Vacca 1997), they need not be physically late-type objects. Instead, they may be earlier-type galaxies that look dramatically different in the rest-frame UV. Because of the wavelength dependence of nearby galaxy morphology (especially towards the UV), faint galaxy classifications will depend on the rest-frame wavelength sampled. This "morphological Kcorrection" can be quite significant, and must be quantified in order to distinguish genuine evolutionary effects from simple band-pass shifting.

Morphological K-corrections were derived from UIT images by Bohlin et al. (1991) and Giavalisco et al. (1996), showing that nearby galaxy morphology can change quite dramatically below 3600-4000 $\AA$, where the hot (young) stellar population - located mainly in spiral arms and H II-regions — dominates the spectral energy distribution (SED) and where bulges essentially disappear (Burgarella et al. 2001; Marcum et al. 2001; Kuchinski et al. 2001). Galaxies 
therefore often appear to be of later Hubble type the further one looks into the rest-frame UV. Qualitatively, this is easy to understand: in the optical/near-IR, we see the accumulated luminous phases of long-lived ( $>1 \mathrm{Gyr}$ ) stars, which emit most of their energy at longer wavelengths, whereas the mid-UV samples the star-formation rate (SFR) averaged over the past Gyr or less. The mid-UV includes the longest wavelengths where young stars can dominate the integrated galaxy light, and traces primarily presently active star forming regions, or those regions where star-formation (SF) has only recently shut down. A number of authors have explored the effects of band-pass shifting using multicolor optical images to extrapolate to the rest-frame UV on a pixel-by-pixel basis using both ground-based data (e.g., Hibbard \& Vacca, 1997; Brinchmann et al. 1998), mid-UV FOCA balloon images (Burgarella et al. 2001), UIT farUV images (Kuchinski et al. 2000), and HST images of galaxies at moderate redshifts (Abraham et al. 1999; Bouwens, Broadhurst, \& Silk 1998). The galaxy shape and size distributions measured in deep HST images appear to considerably exceed the effects of band-pass shifting alone.

To address these issues unambiguously, we present a systematic HST imaging project with the Wide Field and Planetary Camera 2 ("WFPC2") in the mid-UV and red for a representative sample of $\sim 100$ nearby galaxies. A subset was also imaged with the Near-Infrared Camera and Multi-Object Spectrograph ("NICMOS") in H-band to study the older stellar populations, and with the Advanced Camera for Surveys ("ACS") in $H$ - $\alpha$ to trace their recent SF and correlate this with the mid-UV images. This survey will help us better understand the physical drivers of the rest-frame mid-UV emission, i.e., the relation between SF and the global physical characteristics of galaxies, their recent SF history, and the role of dust absorption/scattering. Details are given in Windhorst et al. (2002), Odewahn et al. (2002; \& this Vol.), Eskridge et al. (2003), de Grijs et al. (2003), Jansen (2004; this Vol.), and Taylor et al. (2004).

\section{Strategy, Sample Selection, and HST Observations}

With the HST WFPC2, we imaged $\sim 100$ nearby galaxies through one or, whenever possible, two wide-band mid-UV filters below the atmospheric cutoff: F300W ( $\lambda_{\text {cent }} \simeq 2930 \AA ; \Delta \lambda \simeq 740 \AA$ FWHM $)$ and F255W $\left(\lambda_{\text {cent }} \simeq\right.$ $2550 \AA ; \Delta \lambda \simeq 395 \AA$ FWHM). To this we add short red F814W exposures to provide red-leak removal and study the older stellar population. The F255W, F300W filters are logarithmically about equally spaced in $\lambda$ from Johnson $U$, $B$, and so add significantly to the ground-based optical-near-IR color baseline.

Over the last decade, several groups did systematic ground-based imaging of a large number of nearby galaxies covering all Hubble types and inclinations. Most of these were observed in $U B V R$, but a subset also in the near-IR $I J H K$ filters (Eskridge et al. 2002, see also this volume). We selected our HST sam- 

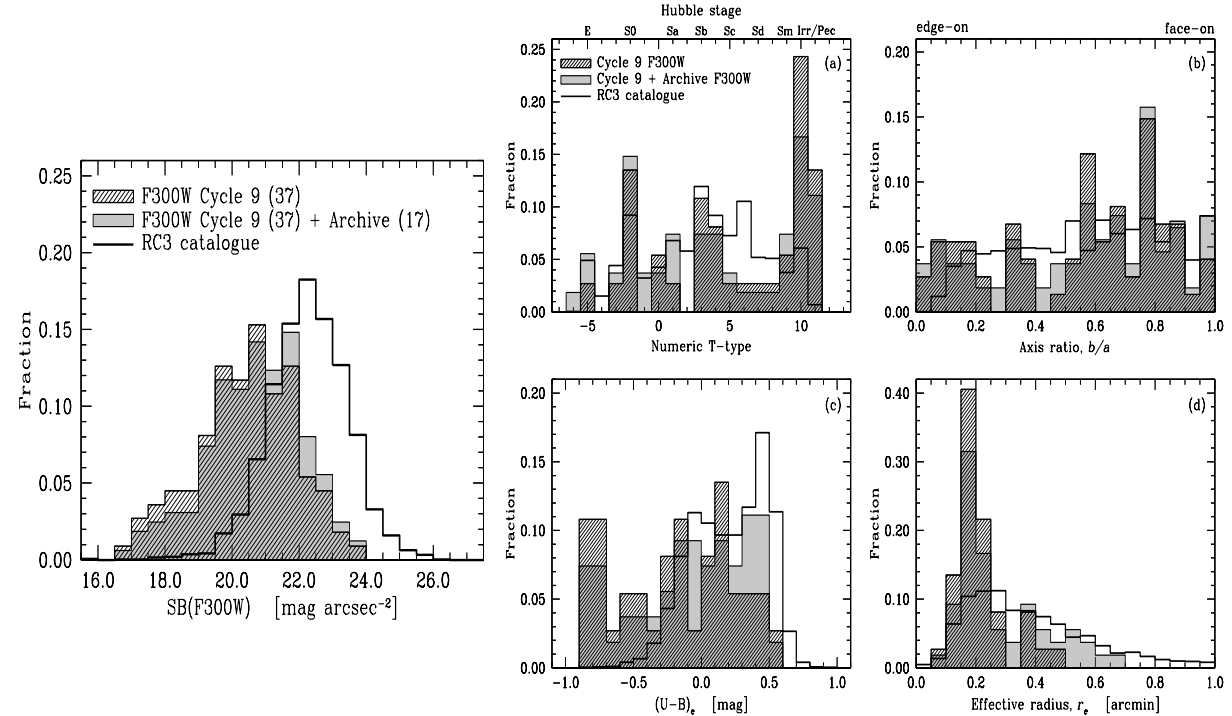

Fig. 1 (LEFT PANEL): The distribution of predicted average mid-UV surface brightness (SB) out to $r_{e}$ for the galaxies observed in HST Cycle 9 (hashed histogram) and for the full galaxy sample (solid histogram), which includes galaxies with mid-UV images in the HST Archive. For comparison, we show the SB-distribution for the 3009 galaxies in the RC3 with measured $B_{T},(U-B)$ and $r_{e}$ (open histogram).

Fig. 2a-2d (RIGHT FOUR PANELS): Other properties of the selected HST mid-UV galaxy sample. As in Fig. 1, we plot the Cycle 9 galaxies (hashed), the full sample (solid), and the RC3 (open) versus (a) morphological type; $(b)$ apparent axis ratio, $b / a ;(c)$ average $(U-B)$ color out to $r_{e}$; and $(d)$ effective radius $r_{e}$. Our sample approximates the RC3 distribution, except that for comparison with high redshift samples we emphasize the later types, bluest galaxy colors, and smallest sizes.

ple of $\sim 100$ galaxies from these ground-based samples, which include: (1) 86 face-on spirals (de Jong \& van der Kruit 1994); (2) 220 spirals from the OSU survey (Eskridge et al. 2000, 2002); (3) 113 galaxies from Frei et al. (1996); (4) 100 galaxies with $1500 \AA$ and 40 with $2500 \AA$ images from the Astro/UIT mission (Kuchinski et al. 2001; Marcum et al. 2001); (5) 48 edge-on galaxies (de Grijs et al. 1997); (6) 150 UGC galaxies that are morphologically irregular, peculiar, or mergers (Hibbard \& Vacca 1997); and (7) 49 late-type dwarf spiral galaxies and compact, high-SB luminous blue galaxies (Matthews \& Gallagher 1997). The last two samples provide likely local counterparts of the peculiar and irregular galaxies seen with $H S T$ in large numbers at high redshifts.

The selection criteria for inclusion in the HST sample are: (a) $B$-band halflight radius 0.1 ' $\lesssim r_{e} \lesssim 1.0$ ', so that the object fits inside a WFPC2 or NIC3 mosaic; (b) predicted average mid-UV SB out to $\mathrm{r} \simeq r_{e}: 18 \sim \mu_{F 300 W} \lesssim 22.5-23.0$ mag $\operatorname{arcsec}^{-2}$ (Fig. 1), so that a galaxy can be detected out to $\mathrm{r} \simeq 2-3 r_{e}$ in one orbit with sufficiently high $\mathrm{S} / \mathrm{N}$ to allow structural features to be recognized; (c) distribution of Hubble types and (d) the distribution over apparent axis ratio (inclination) should both be representative for nearby galaxy samples (Fig. 2). 
Fig. 1 shows that the galaxies observed with $H S T$ have higher predicted mid-UV SB than the median in the RC3, which is $\mu_{F 300 W} \simeq 22.3 \mathrm{mag} \mathrm{arcsec}^{-2}$. This is not an overriding concern, since high-redshift samples are similarly biased (or more so) in favor of high-SB galaxies due to the severe $(1+\mathrm{z})^{4}$ cosmological SB-dimming. Based on Fig. 1, one could apply weights to each observed $H S T$ galaxy to better represent the true local galaxy distribution when complete samples are in order. Figures $2 \mathrm{a}-2 \mathrm{~d}$ show the distributions of the HST mid-UV sample over morphological type, axis ratio $b / a,(\mathrm{U}-\mathrm{B})$ color within $r_{e}$, and effective radius $r_{e}$. Our selected mid-UV sample deliberately overemphasizes late-types, bluer, and smaller galaxies, since these are the dominant galaxy population at high redshifts. Fig. $2 b$ shows that our selected mid-UV galaxies have a similar $b / a$ distribution as the RC3. Since there is no significant trend in the galaxy $b / a$ distribution from the RC3 level $(\mathrm{B} \lesssim 15 \mathrm{mag})$ to the HDF limit ( $\lesssim 28$ mag; Odewahn et al. 1997), our nearby HST comparison sample is thus a fair one in terms of galaxy ellipticities at all redshifts.

In total, $\sim 100$ galaxies were observed with WFPC2 in Cycle 9-10, and about 12 with NICMOS/NIC3 in H-band and 20 with ACS in $H$ - $\alpha$ in Cycle 12 (Jansen 2004; this Vol.). Typically, we exposed 2×800-1000 sec in F300W and $2 \times 100-160 \mathrm{sec}$ in F814W, while some exposures came from the HST Archive. Exposures times were made flexible to optimally use the full HST orbit allocated per galaxy. The resulting 1-orbit SB-sensitivity matches that achieved in deep HST I-band images for faint galaxies, accounting for SBdimming. The resulting point source sensitivity resolves many galaxies into their brightest star-forming regions (OB associations, young star clusters), and resolves the brightest stars in the nearest objects.

\section{Discussion of the HST Images}

In this section, we give a high level discussion of the HST/WFPC2 (Fig. 3) and some of the recent NIC3 images obtained thus far (Fig. 4). The HST images have in general a much higher dynamic range than the ground-based images. This sometimes leads to a different galaxy classification than in the groundbased images or the RC3, and also makes it difficult to properly display the full range of structures seen within a galaxy. The best display of the HST images is given in the electronic version of Windhorst et al. (2002).

- Early-type Galaxies: The regular early-type galaxies (elliptical and S0's) in our sample show in general a significant increase in SB from the mid-UV to the red, reflecting an overall lack of a young stellar population, as also seen in the far-UV UIT images of Marcum et al. (2001) and Kuchinski et al. (2000). Out of 7 early-type galaxies imaged, two have small blue nuclear features. Two galaxies are merger remnants and have significant dusty disks in the mid-UV, but will likely soon evolve into early-type galaxies. Three early-type galaxies 


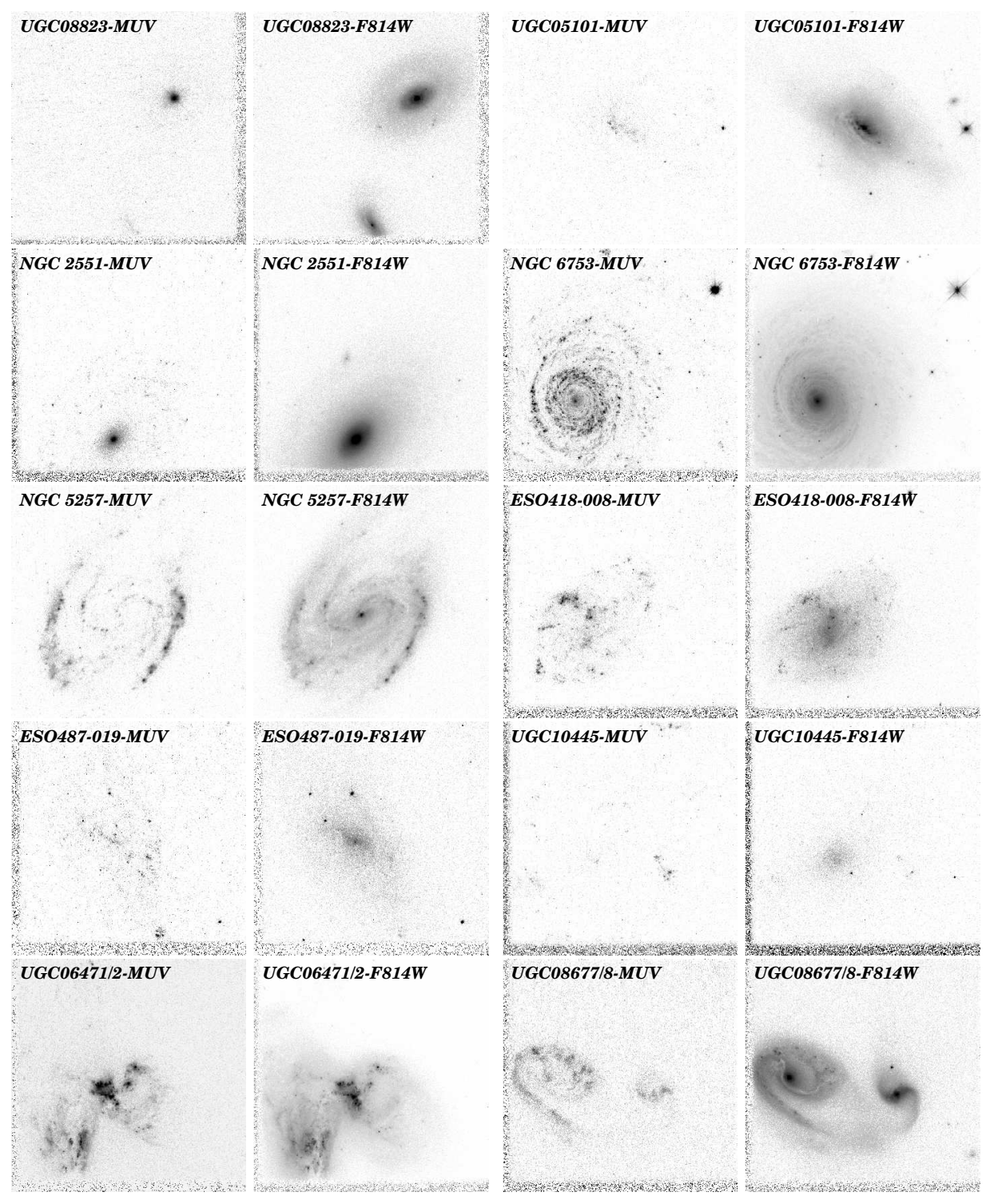

Fig. 3: Summary of our HST/WFPC2-images (75" $\times 75$ " FOV) of nearby galaxies in F300W ("mid-UV"; left panels) and F814W (right panels). (Row 3.1) The Seyfert UGC08823 with an old stellar population in F814W, and the merger remnant UGC05101 which is dusty in the mid-UV; (Row 3.2) The early-type spiral NGC2551 and mid-type grand-design spiral NGC6753; (Row 3.3) The late-type barred spiral NGC5257 and the small spiral ESO418-008; (Row 3.4) The late-types ESO487-019 and UGC10445, each with significant $K_{\text {morph }}$ (which is an exception rather than the rule for the late-type galaxies observed in our sample); (Row 3.5) The mergers UGC06471-2 and UGC08677-8 with various wavelength dependent structures. The best full-size displays are given in the electronic version of Windhorst et al. (2002). 
become dominated by point sources in the mid-UV, indicating weak opticalUV AGN, Seyfert, or LINER nuclei (Fig. 3.1). While these are small number statistics, their presence in our sample is due to our pre-selection of galaxies with high predicted average SB in the mid-UV within $r_{e}$, resulting in a number of early-type galaxies that are dominated in the mid-UV by AGN.

If AGN generally reside in bulge-dominated galaxies (e.g., Magorrian et al. 1998), then the red and old stellar population of the underlying earlytype galaxies will be generally faint in the UV, but the presence of a (weak) AGN will result in blue (U-B) colors and therefore a high SB the mid-near UV, and so inclusion into UV-selected samples. Similarly, ground-based Uor B-band selected surveys result in significant numbers of point-like AGN in early-type galaxies at moderate redshifts $(\mathrm{z} \gtrsim 0.3)$, which are thus classified as QSO's. However, had these objects been selected from the ground at much longer wavelengths (i.e., in the $I$-band), then they would have shown up as early-type galaxies in good seeing. This leads us to wonder to what extent the (strong) cosmological evolution of AGN selected through their UV/blue excess at modest redshifts (Koo \& Kron 1988; Boyle et al. 2000) could in part be due to a "morphological K-correction" of early-type galaxies with weak AGN — the strong SB-dimming of their UV-faint stellar population will render the early-type AGN host galaxies invisible at intermediate to higher redshifts.

- Mid-type Spirals: In general, spiral arms are more pronounced in the midUV, as UIT showed to be generally true in the far-UV (Bohlin et al. 1991, Hill et al. 1992, Kuchinski et al. 2001, Marcum et al. 2001). However, mid-type spirals and star-forming galaxies appear in general more similar from the midUV to the optical than early-type galaxies (Fig. $3.2 \& 3.3$ ). Some appear as later types in the mid-UV, and a few show drastic changes in type from the optical to the mid-UV, equivalent to a change in T-type of $\Delta \mathrm{T} \approx 3$ (Fig. 3.4b). One galaxy shows a spectacular resonance ring of hot stars, while the remainder disk becomes essentially invisible in the mid-UV (Eskridge et al. 2003).

We observe a variation in color of the galaxy bulges/centers in spirals, and a considerable range in scale and SB of the individual star-forming regions. Dust features in mid-type spirals can be well traced by comparing the F300W to the F814W images, and are visible in lanes or patches (possibly trailing spiral density wave patterns?), in pockets, and/or bubbles. A curious feature is that almost without exception, the mid-type spirals in our sample have their small nuclear bulges bisected by a dust-lane, which is often connected to the inner spiral arm structure, as seen by comparing the F814W and mid-UV images. This is interesting in the context of the finding that all bulge-dominated systems have a central black-hole with an average mass $M_{b h} \simeq 0.005 \times M_{b u l g e}$ (Magorrian et al. 1998). The small nuclear dust-lane in most mid-late-type spirals may be involved in feeding the inner accretion disk, if present. Some edge-on galaxies are very faint in F300W when compared to F814W, others 
emit/transmit significantly more in F300W, with some intermediate cases. All show a F300W/F814W flux ratio increasing from the inside out, as expected for a decreasing dust content from the inside out, and/or a strong radial gradient in the stellar population, if we assume that the dust and stars are well mixed (i.e., the stars are not preferentially located in front of most of the dust; see e.g., Jansen et al. 1994, 2000; Witt \& Gordon 1996; Kuchinski et al. 1998).

- Late-type Galaxies and Irregulars: The late-type and irregular galaxies imaged are a heterogeneous mixture. The majority of these galaxies show a F300W morphology that is similar to F814W. Important differences are seen, however, due to recognizable dust-lanes blocking out the F300W light. Dust is visible in pockets, holes or bubbles, perhaps due to supernova-induced outflows, or outflows fueled by bright star-forming regions, such as seen in M82 at $H S T$ resolution (de Grijs et al. 2001). Some late-type galaxies are physically smaller galaxies with what appears to be the beginning of spiral structure. Others are Magellanic Irregulars with various regions of stochastic SF. Star-formation "ridges" are seen in late-type galaxies, as well as hot stars or star-clusters that are particularly conspicuous in the mid-UV (F300W and/or F255W). A few late-type galaxies would be classified significantly differently when observed in the mid-UV than in the F814W passband, especially when observed under less than perfect atmospheric seeing conditions (see Fig. 3.4). A quantitative discussion of the classification changes as a function of restframe wavelength will be given by Taylor et al. (2004).

- Peculiars and Mergers: The majority of the peculiars and interacting galaxies show a F300W morphology that is similar to that in F814W. Several mergers have spectacular dust-lanes that thread along with the spiral arms, which are tidally distorted during the interaction (Fig. 3.5), while most others have absorption in apparently random locations associated with a star-burst.

\section{Galaxy Outskirts from a Cosmological Perspective}

The combined HST+ground-based UBVR light profiles show that early-mid type galaxies have only small radial color-gradients in the outer parts, where disks tend to get somewhat bluer at larger radii (Fig. 4 here; de Jong \& van der Kruit 1994; de Jong 1996; de Grijs et al. 1997; Jansen et al. 2000). Corrected for reddening by dust, this behavior indicates a (thin) disk that grows with cosmic time through the infall of gas and minor mergers that did not recently upset the disk. Remarkably, the majority of the late-type galaxies in our sample shows the opposite behavior, becoming significantly redder at larger radii. Fig. 4a and 4b compare an example of each trend, and in Taylor et al. (2004) we quantify the color-gradients for the total sample. In general, color-gradients 

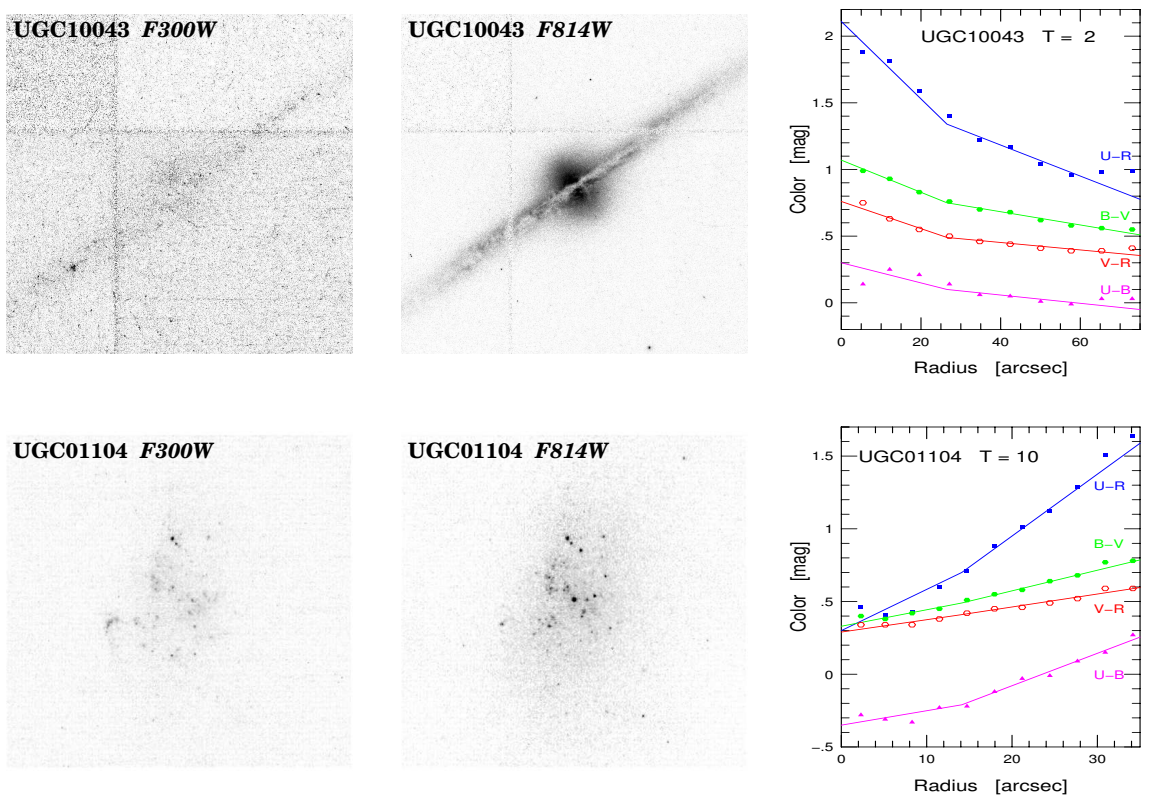

Fig. 4: WFPC2-images in F300W and F814W and color-gradients in (U-B), (B-V), (V-R), and (U-R) for (a) UGC10043 [top] and (b) UGC01104 [bottom]. UGC10043 shows the usual behavior for early-mid-type spirals of getting bluer with increasing radius. Irregular galaxy UGC01104, on the other hand, becomes progressively redder toward its outer regions. This is the more common behavior for late-type/irregular galaxies in our HST samples, and suggest the existence of an old halo or (thick) disk.
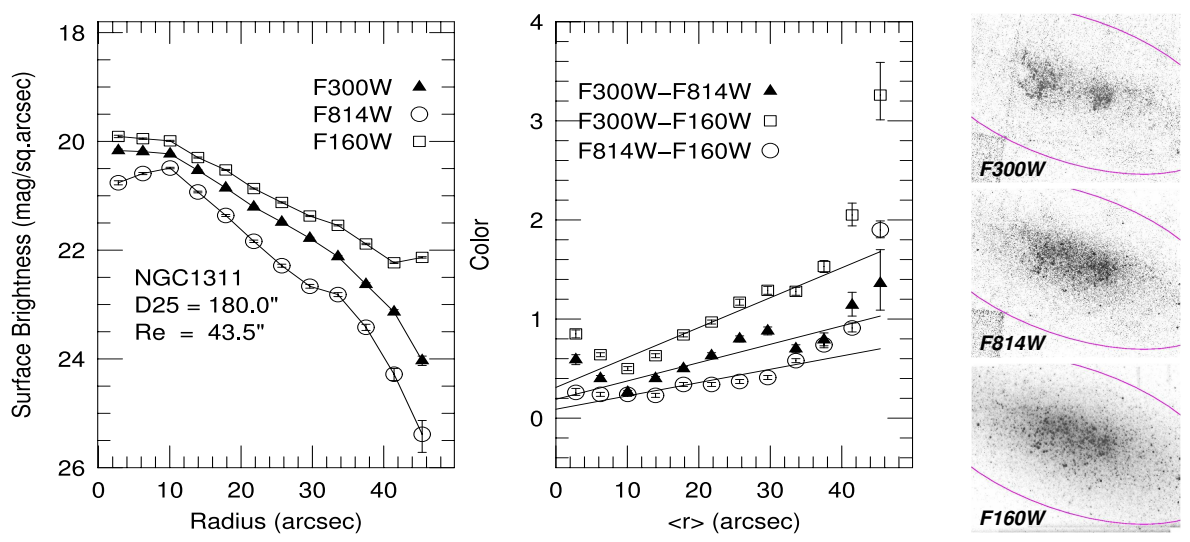

Fig. 5: Profiles of surface brightness [left] and color [middle] measured from our HST/WFPC2 F300W, F814W and NIC3 F160W images [right]. The ellipses in each image correspond to the outermost points used in the profiles. NGC1311 is an Sm galaxy that is well-resolved with HST into its stellar populations from the mid-UV to the near-IR and that becomes redder with increasing distance from its center, a trend seen in the majority of late-type galaxies in our sample. Significantly more stars are seen in H-band (through most of the dust), and a dimmer unresolved, older stellar population is apparent. 
get redder outwards for the majority of late-type galaxies, and this is in general more often seen for galaxies of lower luminosity, smaller half-light radius, and lower average SB (Tully et al. 1996; Jansen et al. 2000; Taylor et al. 2004). This trend appears in all filters, thereby ruling out sky-subtraction errors are the major cause (Fig. 5), and appears somewhat more pronounced for rounder galaxies than for flatter galaxies. While young UV/blue-bright stellar populations clearly dominate their inner morphology, most late-type galaxies thus appear to have a significant old halo or (thick) disk population.

The idea of old Population II envelopes in dwarf irregular galaxies was first proposed by Baade (1958). Tully et al. (1996) found a significant number of nearby dwarf galaxies with red outer envelopes, and Jansen et al. (2000) found a similar result in the NFGS Survey. Recently, Dalcanton \& Bernstein (2002) suggested red envelopes in a sample of late-type edge-on galaxies. These findings constrain hierarchical models of galaxy formation (e.g.Katz et al. 1999; Kauffmann et al. 1999). Dalcanton \& Bernstein (2002) favor a scenario where late-type galaxies formed in an early epoch of significant mergers ( $\gtrsim 6 \mathrm{Gyr}$ ago or $\mathrm{z}_{\sim} 1$ ), resulting in red outer colors, which they explain as an old (thick) disk. These late-type galaxies would have remained largely undisturbed since $\mathrm{z} \simeq 1$, and recently undergone only more centrally concentrated SF.

Important related clues to the nature of late-types can be found from the galaxy counts as a function of type, which are now available over the entire flux range $15 \lesssim \mathrm{B} \lesssim 27 \mathrm{mag}$ (Cohen et al. 2003), so they can be properly normalized at the bright end. At the faint end $(B \gtrsim 24$ mag or $\mathrm{z} \approx 1)$, the type-dependent counts not only show the well-known excess of late-type galaxies, but also show a significant excess of early-mid types at $z \gtrsim 1$. Cohen et al. (2003) explain this as the Cosmological Constant itself gradually turning off the epochdependent merger rate, when it started to significantly affect the expansion at $\mathrm{z} \lesssim 1$. Today's red outskirts in late-type galaxies could thus be the smoking gun of the cosmic accelleration induced by $\Lambda$. As a consequence, most (major) mergers occurred early-on in galaxies of all types and luminosities, so halos and (thick) disks were formed early-on and are still seen as such today. Disks of giant galaxies are still growing today, because of infalling surrounding gas and minor mergers, but are otherwise evolving passively. Late-type

low-luminosity systems are mostly left with stochastic star-formation in their inner parts today, which is no longer fed by their environment in a major way.

\section{Discussion and Conclusions}

In summary, our HST mid-UV imaging data base shows that in galaxies where star-formation is sufficiently pronounced, it can dominate the morphology from the mid-UV though the optical, resulting in very little change in morphology from the UV to the red. However, when stellar populations older than about 1 
Gyr produce most of the optical light, we see changes in morphology between the optical - where these stars tend to produce relatively regular structures due to the effects of orbital mixing within the galaxies - and the mid-UV - where younger stars whose locations still reflect the distribution of their birthplaces. An additional complication is introduced by the presence of dust obscuration. Dust lanes or dust clouds that are nearly transparent in the visible can be opaque in the mid-UV, thereby changing the apparent morphology. In summary, the qualitative results from our study are:

- (1) High-SB early-type galaxies in the optical show a variety of morphologies in the mid-UV that can lead to a different morphological classification, although not necessarily always as later-type. The often rather peculiar midUV morphology of early-type galaxies is generally quite different than that of the real late-type galaxies as seen in the mid-UV.

- (2) About half of the mid-type spirals in the optical appear as later morphological types in the mid-UV, but not all mid-type spiral galaxies do look dramatically different in the mid-UV. Their mid-UV images show a considerable range in the scale and SB of individual star-forming regions. A comparison of F300W to F814W images yields good sensitivity to dust features.

- (3) The majority of the heterogeneous subset of late-type, irregular, peculiar and merging galaxies display mid-UV morphologies that are similar to those seen in the red, but with important differences due to recognizable dustfeatures absorbing the bluer light, and due to hot stars, star-clusters, and starformation "ridges" that are bright in the mid-UV. Less than one third of the galaxies classified as late-type in the optical appears sufficiently different in the mid-UV to result in a different classification.

Our HST mid-UV survey of nearby galaxies shows that - when observed in the rest-frame mid-UV - early- to mid-type galaxies are more likely misclassified as later types than late-type galaxies are misclassified as earlier types. This is because later types are dominated by the same young and hot stars in all filters from the mid-UV to the red, and so have a smaller "morphological K-correction" than true earlier types. The morphological K-correction can thus explain part, but certainly not all of the excess faint blue late-type galaxies seen in deep HST fields. Classification of faint galaxies in the rest-frame mid-UV will likely result in some fraction of early-mid type galaxies being misclassified as later-types, likely a larger fraction than vice versa.

In conclusion, it is unlikely that the morphological K-correction can explain all of the faint blue galaxy excess as misclassified earlier-type galaxies. And the morphological K-correction cannot explain the slight excess of earlymid type galaxies at faint magnitudes ( $B \gtrsim 24 \mathrm{mag}$ ) with respect to passively evolving models, as found by Odewahn et al. (1996) and Cohen et al. (2003), since the main misclassification error goes in the opposite direction, as discussed above. Instead, our mid-UV survey seems to support the conclusion 
of Cohen et al. (2003) that the number of faint galaxies is larger than the nonevolving predictions for all galaxy types, but more significantly so for the later types. They give a possible explanation of this finding in terms of hierarchical formation models, where the Cosmological Constant itself was responsible for gradually turning off the strongly epoch-dependent merger rate at $\mathrm{z} \lesssim 1$, since in WMAP cosmology the universe became dominated by $\Lambda$ and started expanding exponentially for $\mathrm{z} \lesssim 0.4$. Hence, $\Lambda$ may be responsible for the gradual transition from the $\mathrm{z} \approx 1$ Universe, where galaxy formation and evolution was driven by major mergers, to the Universe at $\mathrm{z} \lesssim 0.4$, where at best minor mergers or infall affected the more passive, secular evolution of giant galaxies.

\section{Acknowledgments}

We acknowledge support from NASA grants GO-8645.*, GO-9124.*, GO9824.*, GO-9892.*, and AR-8765.*, awarded by STScI, which is operated by AURA for NASA under contract NAS 5-26555.

\section{References}

Abraham, R. G., et al. 1996, MNRAS, 279, L47

Abraham, R. G., et al. 1999, MNRAS, 308, 569

Baade 1958, AR 5, 303

Beckwith, S. V. W., et al. 2004, in prep.

Bohlin, R. C., et al. 1991, ApJ, 368, 12

Bouwens, R. J., et al. J. 1998, ApJ, 506, 579

Boyle, B. J., et al. 2000, MNRAS, 317, 1014

Burgarella, D., et al. 2001, A\&A, 369, 421

Cohen, S., et al. 2003, AJ, 125, 1762

Conselice, C. J., et al. 2000, AJ, 119, 79

Cowie, L. L., et al. 1995, Nature, 377, 603

Dalcanton \& Bernstein 2002, AJ 124, 1328

de Grijs, R., \& Peletier, R. F. 1997, A\&A, 320, L21

de Grijs R., et al. 2001, AJ, 121, 768

de Grijs R., et al. 2003, MNRAS, 342, 259

de Jong, R. S., et al. 1994, A\&ApS, 106, 451

de Vaucouleurs, G., et al. 1991, 3rd Reference Cata-

logue of Bright Galaxies (New York: Springer)

Driver, S. P., et al. 1995a, ApJ, 449, L23

Driver, S. P., et al. 1995b, ApJ, 453, 48

Driver, S. P., et al. 1998, ApJ, 496, L93

Ellis, R.S., et al. 1996, MNRAS, 280, 235

Eskridge, P. B., et al. 2003, ApJ586, 923

Eskridge, P. B., et al. 2002, ApJS 143, 73

Giavalisco, M., et al. 1996, AJ, 112, 369

Glazebrook, K., et al. 1995, MNRAS, 275, L19

Hibbard, J. E., et al. 1997, AJ, 114, 1741

Hill, J. K. et al. 1992, ApJ, 395, L37
Jansen, R. A., et al. 1994, MNRAS, 270, 373

Jansen, R. A., et al. 2000a, ApJS, 126, 271

Katz et al. 1999, ApJ 523, 463

Kauffmann et al. 1999, MNRAS 303, 188

Koo, D. C., \& Kron, R. G. 1988, ApJ, 325, 92

Kuchinski, L. E., et al. 1998, AJ, 115, 1438

Kuchinski, L. E., et al. 2001, AJ, 122, 729

Lilly, S. J., et al. 1998, ApJ, 500, 75

Marcum, P. M., et al. 2001, ApJS, 132, 129

Matthews, L. D. et al. 1997, AJ, 114, 1899

Odewahn, S. C., et al. 1996, ApJ, 472, L013

Odewahn, S. C., et al. 1997, AJ, 114, 2219

Odewahn, S. C., et al. 2002, ApJ, 568, 539

Pascarelle, S. M., et al. 1996b, Nature, 383, 45

Roche, N., et al. 1997, MNRAS, 288, 220

Taylor, V. A., et al. 2004, AJ, in preparation

Williams, R. E., et al. 1996, AJ, 112, 1335

Windhorst, R. A., et al. 1997, in "The Ultraviolet Universe at Low and High Redshift", Ed. W. H. Waller, et al. (New York: AIP Press), Vol. 408, 242

Windhorst, R. A., et al. 2000, in "Toward a New Millennium in Galaxy Morphology", Ed. D. Block et al. Astroph. and Space Sc., Vol. 269-270, 243

Windhorst, R. A., Taylor, V. A., Jansen, R. A., Odewahn, S. C., Chiarenza, C. A., Conselice, C. J., de Grijs, R., de Jong, R. S., MacKenty, J. W., Eskridge, P. B., Frogel, J. A., Gallagher III, J. S., Hibbard, J. E., Matthews, L. D., \& O'Connell, R. W. 2002, ApJS, 143, 113 ("the HST mid-UV team")

Witt, A. N., \& Gordon, K. D. 1996, ApJ, 463, 681 